\title{
Contribuição ao estudo das Gregarinas (1)
}

por

CHESAR PINTIO, D. M.

(Com as estampas 1-6).

Introdução.

O presente trabalho representa 0 resultado de pesquizas que encetámos em Setembro de 1917, sob a orientação dos DRS. OLYMPIO DA FONSECA FILHO $\mathrm{E}$ ARISTIDES MARQUES DA CUNHA, Assistentes do Instituto Oswaldo Cruz.

Em doze Notas-prévias que publicamos no BRASIL-MEDICO durante o ano de 1918, descrevemos 16 especies novas de gregarinas além de termos estabelecido dois generos novos. Num deles incluimos uma especie dentre as novas fórmas que descrevemos, estabelecendo outro para uma gregarina já anteriormente conhecida. Das gregarinas conhecidas damos a redescrição de duas delas por havermos observado alguns detalhes não referidos pelos autores que as descreveram.

Os artrópodes por nós examinados atingiram o numero de 471 , todos eles colhidos no Rio de Janeiro (Léme e Man- guinhos), em diferentes epocas do ano. Dos miriapodes colhidos e examinados pelo DR. OLYMPIO DA FONSECA FILHO quando em viagem scientifica pelo rio PARANA' (Estado de São Paulo), verificámos sómente duas especies de gregarinas já conhecidas: STENOPHO. RA JULI e STENOPHORA COCKERELLAE.

Só conseguimos verificar o ciclo evolutivo de uma gregarina depois de dez mezes de pesquisas, pois os esporos destes protozoarios eram sempre rarissimos, embora tivessemos examinado artrópodes em todas as estações do ano. O unico animal que apresentou grande numero de esporos foi um oligoqueto (GLOSSOSCOLEX WIENGREENI) apanhado pelo DR. O. DA FONSECA FILHO nos arredores do Rio de Janeiro (GAVEA); aproveitando então o grande numero de esporos do MONOCYSTIS PERFORANS Pinto. 1918 existente no tecido parasitado (testiculo) cavidade geral e nas fezes,

(1) Trabalho laureado pela Faculdade de Medicina do Rio de Janeiro com o premio "Gunning" de Historia Natural (1919). 
resolvemos observar a formação dos esporozoitos in vitro, o que conseguimos com relativa facilidade.

\section{Mistorico brasileiro.}

Sobre o assunto presente só conhecemos um trabalho brasileiro, do Professor DR. PEDRO SEVERIANO DE MAGALHãES, publicado nos "Archives de Parasitologie» de Paris. em 1900. Actualmente o Professor DR. GUSTAVO HASSELMANN, da Escola de Agricultura, publicou em o BRASIL-MEDICO cinco notas-previas sobre esporozoarios e descreveu algumas especies novas de gregarinas além de ter estudado muito bem o ciclo evolutivo destes protozoarios.

\section{Tecnica.}

OBTENÇÃO DE MATERIAL PARA ESTUDOS. - Entre os invertebrados que mais frequentemente são parasitados pelas gregarinas, estão os artrópodes, anelideos, moluscos, equinodermes etc. Dos artrópodes, o mais facil de obtenção é a barata STYLOPYGA AMERICANA que entre nós é parasitada pelas seguintes especies: GREGARINA BLATTARUM von Siebold, PROTOMAGALHAENSIA SERPENTULA (MAGALHĀES, 1900) e GREGARINA LEGERI Pinto, 1918.

Uma vez morto o artrópode, molusco etc. pelo cloroformio ou eter retira-se o intestino que deve ser aberto longitudinalmente por meio de uma pequena tezoura que possua as extremidades bem ponteagudas. Feito isto, podemos muitas vezes observar a olho nú, pequenos bastonetes de côr branca; com uma pipeta aspira-se o conteudo intestinal juntamente com os pequenos bastonetes brancos e examina-se ao microscopio com pequeno aumento (Obj. A. ZEISS). Verificada a presença de gregarinas no intestino do animal morto, faz-se a fixação do orgão parasitado para ulteriormente inclui-lo em parafina afim de praticarem- se córtes histologicos. Nestes córtes então, iremos procurar as fórmas endocelulares das gregarinas.

\section{Preparados.}

Os preparados pódem ser a fresco ou corados. Os preparados corados fizemo-los pelo metodo de HEIDENHAIN, hematoxilina de DELAFILD ou hematoxilina de HANSEN. Os cótes de intestino contendo fórmas endocelulares foram igualmente coloridos pelo metodo de HEIDENHAIN que sempre deu bons resultados.

Para os estudos de sistematica será melhor desenhar o protozoario quando vivo, pois com a fixação e coloração muitas vezes as gregarinas se fragmentam devido as dimensões exageradas que elas apresentam.

\section{Estudo dos generos e especies brasi- leiras.}

Durante nossas pesquisas sobre esporozoarios do Brasil verificámos a existencia de seis generos de gregarinas, sendo que dois deles foram por nós estabelecidos. No estudo sistematico de algumas especies, encontrámos certas dificuldades para coloca-las em generos já conhecidos, e como não possuisemos base scientifica para crear novos generos colocámos provisoriamente algumas especies no genero GREGARINA, DUFOUR até que estudos posteriores elucidem melhor a classificação daquelas especies.

\section{Gregarinas policistides (Cefalinas).}

I. Fam. STENOPHORIDAE (LEGER et DUBOSCQ, 1904.) PINTO, 1918.

a) Genero STENOPHORA LABBE'. 1899.

b) Genero FONSECAIA PINTO, 1918.

II. Fam. GREGARINIDAE LABBE', 1899.

a) Genero GREGARINA DUFOUR, 1828 . 
b) Genero PROTOMAGALHAENSIA PINTO, 1918.

III. Fam. ACTINOCEPHALIDAE LEGER, 1892.

a) Genero BOTHRIOPSIS SCHNEIDER, 1875.

\section{Gregarinas monocistides (Acefalinas).}

I. Fam. MONOCYSTIDAE BUTSCHLI, 1822.

a) Genero MONOCYSTIS STEIN. 1848

\section{Genero Fonsecaia PINTO, 1918.}

Diagnose do genero.-Gregarina policistide com desenvolvimento intra celular, não formando sizigia. Esporocistos lisos em fórma de elipse alongada sem linha equatorial. não reunidos em cadeia e desprovidos de endo-esporocisto. Epimerito muito redusido e desprovido de endoplasma. Gregarina parasita de diplópode. Cistos, desconhecidos.

Este genero é incluido na familia das STENOPRORIDAE LEGER et DU. BOSCQ, 1904. cuja diagnose modificámos, nela incluindo especies com esporocistos desprovidos de linha equatorial e sem endo-esporocisto.

\section{Especie typo do genero: Fonsecaia polymorpha PINTO, 1918.}

(Est. 1 Rigs. 1-17).

Dimensões dos exemplares.-Fórma redonda (Figs. 2 e 3 ). Comprimento total 30 micra; comprimento do protomerito 8 micra; largura do mesmo 11 micra; idem do deutomerito 24 micra.

Fórma intermediaria (Figs. $4-9$ ). Comprimento total 100 micra; idem do protomerito 10 micra; largura do mesmo 14 micra; idem do deutomerito 48 micra.

Esporonte (Fig. 17). Comprimento total 170 micra; idem do protomerito 15 micra; largura do mesmo 18 micra; idem do deutomerito 80 micra. Nucleo com 10 micra; cariosoma com 3 micra.

Dimensões do espóro. - (Fig. 1). Comprimento 18 micra por 8 micra de largura. Relações entre as dimensões do esporonte (Fig. 17); relação entre o comprimento do protomerito e o comprimento total da gregarina $1: 11,3$. Relação entre a largura do protomerito e a do deutomerito 1:4,4. Relação entre a largura do protomerito para o comprimento do mesmo $1: 1,2$.

Descricão.-A Fonsecaia polymorpha se apresenta sob duas fórmas distinctas, entre as quais existem tipos intermediarios. São elas: fórma redonda (Figs. 2 e 3) aparentemente desprovida de epimerito, e forma longa (Figs. 4-17) com epimerito quasi constante, mas algumas vezes rudimentar.

Fórma redonda- - (Figs. 2 e 3). Gregarina com 30 micra de diametro apresentando um protomerito hemisferico com 8 micra de compriniento por 11 micra de largura. Deutomerito quasi sempre completamente esferico com epicito bastante desenvolvido; mede o deutomerito cerca de 24 micra de diametro. O septo é plano.

Fórma longa. - (Figs. 4-17). Epimerito rudimentar em alguns exemplares e, além disso, faltando algumas vezes; esta parte da gregarina é sempre desprovida de endoplasma. $O$ protomerito tem a fórma de cilindro achatado ou, ainda, pirifórme. O septo é ás vezes invisivel. Deutomerito de morfologia bastante variavel nas fórmas longas do parasito. Em alguns exemplares é cilindrico, podendo terminar numa ponta que é sempre romba. Em outros exemplares á parte media do deutomerito correspondo uma dilatação que se vae atenuando para as duas extremidades; essa dilatação algumas vezes em lugar de se localisar na parte média do deutomerito, se desloca em direção a uma das extremidades, ficando nesses casos uma das porções estreitadas mais alongada que a 
outra, de modo a simular o parasito um vaso provido de gargalo (Est. 1 Fig. 12).

Além dessas variações individuais da fórma do deutomerito, póde um mesmo exemplar de gregarina, em virtude de uma contração anular dos mionemas, apresentar uma especie de cintura que se desloca ora num, ora noutro sentido. (Est. 1 Fig. 7). Nos esporontes que observámos, o deutomerito se apresentava com fórma ovoide (Est. 1 Fig. 17). Vimos um esporonte com essa fórma que gradativamente foi se contraindo até tomar a fórma de uma pera (Est. 1 Fig. 11) deixando ver na base, o protomerito de tamanho muito reduzido.

Epicito bastante espesso em quazi todos os exemplares. Fibrilas de mionema ora invisiveis nas partes anterior e média do deutomerito. em toda a extensão dele. Endocito de coloração castanha ou amarelada, provido de granulações sempre mais condensadas no deutomerito. Em um exemplar notámos nitido afastamento dessas granulações da parte média do deutomerito. na qual se formava uma zona equatorial em que eram apenas visiveis as fibrilas de mionema (Est. 1 Fig. 17). As granulações do deutomerito podem se aglomerar na parte central dele. deixando assim um espaço hialino nas imediaçðes do epicito (Est̂. 1 Fig. $9 \mathrm{~A}$ ).

Nucleo. Esta parte do protozoario tambem aparece sob diversos aspétos. Em algumas gregarinas o nucleo é invisivel a fresco. Em outras se póde apresentar redondo, mostrando ou não, cariosoma esferico e de tamanho variavel, visivel a fresco. O nucleo é as vezes alongado, em fórma de rosacea (Est. 1 Fig. 13) ou de pera (Est. 1 Fig. 14).

Movimentos.-Nas fórmas redondas e nos esporontes não observámos movimentos; não se dando o mesmo nas gregarinas de tamanho médio, onde notámos movimentos ora lentos, ora exagerados. A gregarina se move sempre em direção paralela ao seu eixo longitudinal, fazendo geralmente um pequeno percurso para diante e lógo em seguida outro para traz. Nas fórmas de tamanho médio e que possuem mionemas, notámos que a gregarina se dobrava ao meio e se deslocava para um e outro lado. Tais movimentos nunca observámos nos esporontes que eram quasi imoveis.

Cistos.-Não conseguimos observa-los apezar de termos examinado grande numero de diplópodes.

Esporocistos.-Já descritos.

Procedencia do hospedador: Hospital Central do Exercito. Jockey-Club. Rio. Epoca: Abril de 1918.

Hospedador: ORTHOMORPHA GRACILIS C. Koch, 1847.

Habitat: intestino.

\section{Stenophora cunhai PINTO, 1918.} (Est. 6 Figs. 87 e 88).

Dimensões da gregarina: comprimento total 250 micra; idem do protomerito 50 micra; largura do mesmo 40 micra; idem do deutomerito 30 micra.

Nucleo com 20 micra e cariosoma 10 micra.

Relaç̃es entre essas dimensð̃es: relação entre o comprimento do protomerito para o comprimento do mesmo 1:1,2.

Protomerito apresentando uma parte anterior globulosa seguida de um estreitamento anular. Para traz desse estreitamento existe uma nova porção dilatada seguida de novo estreitamento que corresponde ao septo que o separa do deutomerito. Na parte anterior do protomerito existe uma serie de denticulaçóes.

Deutomerito cilindro-curvo, apresentando na parte anterior uma dilatação semelhante á que se encontra na parte posterior do protomerito. Epicito de espessura unifórme. Fibrilas de mionema muito desenvolvidas, formando uma estriação longitudinal facilmente visivel. Endocito claro, hialino com poucas granulações nas extremidades anterior e posterior.

Nucleo redondo apresentando um 
grande cariosomo central e tendo na superficie uma massa de cromatina com a fórma semi-lunar.

Hospedador: RHINOCRICUS PUGIO Brölemann, 1902. (Gongolo).

Habitat: intestino.

Procedencia do hospedador: Rio de Janeiro (Léme).

Epoca: novembro de 1917.

Stenophora lutzi PINTO, 1918.

(Ests. 2, 5 e 6 Figs. 32-36, 82-85)

Dimensões do protozoario.--Oservámos exemplares livres na cavidade intestinal do hospedador que mediam desde 15 micra até 210 micra. Comprimento total do esporonte 210 micra; idem do protomerito, 28 micra; largura do protomerito na parte posterior 28 micra; idem do deutomerito 35 micra; comprimento do epimerito 5 micra; largura do mesmo 8 micra.

Relações entre tais dimensões.-Relação entre o comprimento do protomerito e o comprimento total da gregarina 1:7,5. Relação entre a largura do deutomerito e a do protomerito 1:1,2. Relacão entre a largura do protomerito e o comprimento do mesmo 1:1.

Epimerito hemisferico, constante nas fórmas intermediarias e ausente na mór parte dos esporontes.

Protomerito cilindrico, possuindo sempre uma cintura na parte média; ás vezes nota-se na parte anterior um vestigio do epimerito que se destacou.

Deutomerito cilindrico, termilando em ponta romba. Epicito flexivel e de espessura uniforme. Endocito de côr parda, granuloso no protomerito e no deutomerito; aqui ás vezes ele é mais escuro na parte anterior.

Nucleo redondo, quasi sempre colocado na parte posterior do protista, possuindo um cariosoma redondo e central. Esporos e cistos desconhecidos.

Diagnose diferencial. A presente gregarina assemelha-se á STENOPHORA DAUPHINIA WATSON, dela se distin- guindo por não possuir póro e papilas na parte anterior do protomerito. WATSON não se refere á existencia do epimerito na gregarina ácima citada, o que se observa em exemplares de STENOPHORA LUTZI.

Hospedador: Rhinocricus (sp.).

Habitat: intestino.

Procedencia: Rio de Janeiro (Léme).

Epoca: Novembro de 1917.

\section{Stenophora cruzi PINTO, 1918.} (Est. 6 Figs. 89.)

Comprimento total da gregarina 400 micra; idem do protomerito 30 micra; largura do protomerito 40 micra; do deutomerito 80 micra. Nucleo com 10 micra.

Relações entre essas dimensões: relação entre o comprimento do protomerito e o comprimento total $1: 13$; relação entre a largura do deutomerito para o comprimento do mesmo $1: 1,3$.

Protomerito com a fórma de cone truncado. Deutomerito cilindrico em a parte anterior estreitando-se na parte posterior para terminar em ponta romba. Esta gregarina apresenta sempre o deutomerito recurvado, Epicito de espessura uniforme deixando transparecer nitidas fibrilas de mionema. Em alguns pontos existem dobras de tamanho variavel. dando assim a impressão de sulcos. Endocito claro hialino, cheio de pequenas granulações. Nos lados e na extremidade posterior $o$ endocito apresenta-se com a fórma vacuolar bastante pronunciada. No protomerito existem granulações, não havendo entretanto fibrilas de mionema. Nucleo pequeno e redondo.

Habitat: intestino de Rhinocricus (sp.).

Procedencia: Rio de Janeiro. (Léme). Epoca: Dezembro de 1917.

\section{Stenophora viannai PINTO, 1918. \\ (Est. 6 Figs. 90-92.)}

Comprimento total do portista 900 micra a 1.000 micra; idem do p'otomerito $60 \mathrm{micra}$; largura do protomerito $70 \mathrm{mi}$ - 
cra; idem do deutomerito 150 micra; epimerito 10 micra por 30 micra. Nucleo 30 micra.

Relaç̃es entre tais dimensões: relação entre o comprimento do protomerito e o comprimento total $1: 16,6$; relação entre a largura do deutomerito e a do protomerito $1: 2,1$; relação entre a largura do protomerito para o comprimento do mesmo 1:1.1.

Epimerito. Na parte anterior do protomerito existe o epimerito que tem a fórma de uma saliencia mais ou menos cilindrica com um póro central. Protomerito heisferico com septo bastante nitido. Deutomerito cilindrico terminando em ponta romba. Esta gregarina tem um polimorfismo bastante pronunciado. Em alguns exemplares o deutomerito afina-se no terço superior; não se dando o mesmo com o protomerito que conserva quasi sempre a mesma morfologia. Nucleo. Esta parte do protista chama lógo a atenção do observador quer pela sua morfologia quer pela situação. Ele é sempre de fórma alongada, colocado transversalmente na maior parte das vezes junto ou nas imediaç̃es do deutomerito. Epicito de espessura variavel. Nos bordos do septo é de espessura bastante pronunciada e mais ainda nos bordos do póro do epimerito. Endocito granuloso, de côr parda no deutomerito e sempre claro no protomerito.

Hospedador: Rhinocricus (sp.).

Habifat: intestino.

Procedencia: Rio de Janeiro (Léme). Epoca: Fevereiro de 1918.

\section{Stenophora polydesmi (LANKESTER,} 1863) WATSON, 1916. (Est. 3 Fig. 49).

Sinonimia :

Gregarina polydesmi virginiensis LEIDY, 1853.

Gregarina polydesmi LANKASTER. 1863.

Amphoroides polydesmi LABBE', 1889.
Gregarina polydesmivirginiensis CPAWLEY, 1903.

Amphoroides polydesmivirginiensis ELLIS, 1913.

WATSON redescreveu esta especie de Stenophora em 1916 e dá para os esporontes 400-900 micra de comprimento. Dimensões dadas por nós: comprimento total 370 micra; largura do deutomerito 40 micra; idem do protomerito 30 micra; comprimento do mesmo 40 micra. $\mathrm{Nu}$ cleo com 10 micra de diametro.

Nas estampas dadas por WATSON não se vem bem certos detalhes sobre a constituição desta gregarina, por isso resolvemos dar uma figura desenhada ad naturalis.

A Stenophora polydesmi (Lank.) WAT., possue um epimerito disposto em fórma de calote, sem constituição granulosa. O protomerito é granuloso e possue mionemas dirigidos no sentido longitudinal do protozoario, sendo de notar que nesta parte do protista não vimos nunca mionemas transversais. O septo é claro e um pouco concavo, estando a concavidade voltada para a extremidade anterior. O deutomerito que é bastante longo, possue granulações de côr parda em toda a sua extensão. Tambem no deutomerito existem mionemas que se dirigem no sentido longitudinal. O epicito desta Stenophora possue nitidos mionemas que se dirigem no sentido transversal. Estas formações, porém. são vistas sómente na periferia do protista.

Hospedador: Rhinocricus (sp.)

Habitat: intestino.

Procedencia: Rio de Janeiro. (Léme). Epoca: Novembro de 1917.

\section{Stenophora umbilicata PINTO, 1918.} (Est. 5 Pigs. 70 c 80 ).

Comprimento total do esporonte, 320 micra; comprimento do protomerito, 25 micra; largura do deutomerito, 150 micra; idem do protomerito, 40 micra; nucleo com 40 micra, cariosoma com 10 micra. Epimerito rudimentar. Protomerito 
muito pequeno e fortemente achatado. Deutomerito muitissimo largo em relaça ao protomerito, de contorno eliptico.

o conjunto formado pelo epimerito * protomerito, fortemente achatados. dá $§$ estremidade anterior da gregarina um aspéto umbilicado. Epicito não diferenciado em alguns esporontes, em outros ele se esboça na parte anterior do deutomerito. Nucleo muito grande, de fórma arredondada, possuindo no interior um grande cariosoma esferico e centralmente colocado.

Hospedador: Rhinocricus (sp.)

Habitat: intestino.

Procedencia: Rio de Janeiro. (Léme).

Epoca: Dezembro de 1917.

\section{Stenophora tenuicollis PINTO, 1918.}

(Est. 5 Fig. 81.)

Comprimento total do esporonte, 400 micra; idem do protomerito, 20 micra: largura do mesmo, 20 micra; idem do deutomerito na parte mais dilatada, 50 micra; idem na parte mais estreita, 18 micra. Nucleo com 10 micra de diametro. Protomerito em fórma de cone. Deutomerito recurvado e apresentando diametro variavel em toda a sua extensão. Na extremidade anterior ele apresenta uma forte dilatação que dá á esta parte do corpo a fórma de um balão; para traz o deutomerito se continua por uma parte estreitada que se vae gradualmente alongando até a extremidade posterior dilatada em fórma de clava. Entre as duas extremidades dilatadas do deutomerito, a parte estreitada tem um aspéto de gargalo fino $\mathrm{e}$ alongado. $\mathrm{O}$ endocito é claro no protomerito e no deutomerito. Esta gregarina possue mionemas altamente desenvolvidos no protomerito e na parte média do deutomerito.

Hospedador: Rhinocricus (sp.)

Habitat: intestino.

Epoca: Setembro de 1917. neiro.

\section{Genero Protomagalhaensia PINTO, 1918.}

Diagnose do genero: espóros em f6rma de barril com um espinho em cadr angulo. Evolução das gregarinas sempre intra-celular. Cistos desconhecidos. Fórmas em evolução sempre alongadas. Sizigia: protomerito do satelite comprimindo á maneira de uma tenaz o deutomerito do primito. Epimerito desconhecido. Este genero é incluido na familia Gregarinidae LABBE', 1889.

Apresentamos um quadro demonstrativo dos caracteres diferenciais entre o presente genero e o genero Gregarina DUFOUR. 1828.

Genero Gregarina DUFOUR, 1828.

Espóro: em fórma de barril ou cílindrico.

Evolução: intra-celular.

Cistos: com espóro-ductos.

Fórmas em evolução: alongadas arredondadas.

Epimerito: globular ou cilindrico.

Sizigia: protomerito do satelite simplesmente aderente ao deutomerito do primito.

Genero Protomagalhaensia PINTO. 1918.

Espóro: em fórma de barril com uñ espinho em cada angulo.

Evolução intra-celular.

Cistos: desconhecidos.

Fórmas em evolução: sempre alongadas.

Epimerito: desconhecido.

Sizigia: protomerito do satelite comprimindo á maneira de uma tenaz o deutomerito do primito.

Especie tipo do geneo: Protomagalhaensi serpentula (MAGALHÃES, 1900) PINTO, 1918.

(Ests. 3 e 6 Figrs. 48 e 93)

Sinonimia :

Gregarina serpentula MAOALHÃES. 1900. 
Dimensóes dadas pelo Professor MAGALHAES : exemplares de $1,2 \mathrm{~mm}$ de comprimento por $0,18 \mathrm{~mm}$ de largura, ordinariamente medem eles $0,77 \mathrm{~mm}$ a 0,80 mm de comprimento por $0,06 \mathrm{~mm}$ de largura.

O protomerito tem 50 micra de comprimento.

A Protomagalhaensia serpentula (MAGALHAES, 1900) apresenta sempre a fórma cilindrica e bastante alongada possuindo mionemas muito nitidos em quasi todos os exemplares por nos examinados; tais formações são vistas no protomerito e no deutomerito do protista, dirigindo-se elas no sentido longitudinal da gregarina. Não observámos nunca mionemas transversais no epicito desta gregarina.

Em alguns exemplares notámos uma pequena mancha parda e de fórma oval, localisada no protomerito dos esporontes. Tambem no protomerito deste protista observa-se em sua parte média uma pequena cintura motivada pelos movimentos que a parte anterior do protomerito efétua para um lado e outro do animal. As sizigias podem ser em Y ou forquilha; sendo de notar que neste estado, os satelites tem modo diverso de se prenderem ao primito. $O$ satelite verdadeiro prende-se ao primito comprirnindo-o em sua parte posterior á maneira de uma tenaz (Fig. 48); o falso satelite tem modo de apreensão diferente do primeiro, isto é, prende-se ao primito como o satelite da Gregarina aragãoi PINTO, 1918. barata).

Habitat: intestino.

Procedencia: Rio (Léme).

\section{Gregarina magalhãesi PINTO, 1918.} (Ests. 1 e 2 Figs. 18-29.)

Dimensṍes da fórma joven do proIista: comprimento total 80 micra; comprimento do protomerito 20 micra; largura do mesmo 20 micra; idem do deutomerito 25 micra; comprimento do epi- merito 10 micra; largura do mesmo 8 micra. Nucleo 10 micra. Dimensões do esporonte: comprimento total 300 micra; idem do protomerito 65 micra; largura do mesmo 60 micra; idem do deutomerito 70 micra.

Epimerito. (Figrs. 18-22 e 24). Esta parte da Gregarina magalhaesi é bastante movel. dando lugar assim a diversas fórmas de epimerito. Quando em repouso, ele tem a fórma de um cone. (Fig19) e é sempre desprovido de granulaç̃es.

Protomerito apresentando um pleomorfismo bastante notavel; nas fórmas jovens do protozoario (Fig. 24) ele possue a fórma cilindro-conica, outras vezes o protomerito se nos apresenta acha. tado no sentido antero posterior (Fig. 23); em alguns exemplares observámos protomeritos tambem achatados e com saliencias e reintrancias, ora na parte anterior (Fig. 25) ora nos bordos (Fig. 26). Esta mudança de fórma do protomerito é devida a movimentos efetuados pela gregarina, deslocando-se o protomerito para um lado e outro. Septo plano ou recurvado ligeiramente, podendo tambem deixar de ser visto algumas vezes.

Deutomerito cilindrico, terminando em ponta romba nas gregarinas jovens: nas fórmas intermediarias (Fig. 29) o deutomerito apresenta mais ou menos a Pórma de um S. Quando a gregarina se desloca para a frente, o deutomerito vae se dobrando aos poucos e toma então a fórma de um $\mathrm{S}$. achatado.

Epicito podendo ser invisivel em atguns exemplares (Fig. 26 e 28), outras vezes ele é espesso e fórma dobras em grande numero e de tamanho variaveis, no deutomerito (Fig. 29). Algumas vezes o epicito penetra em dois terços do deutomerito, dando deste modo a impressão de sulcos (Fig. 29). Endocito claro com granulações no protomerito; no deutomerito ele é bastante escuro, apresentando tambem numerosas granulações. Nuclea 
de tamanho variavel e de morfologia diversa.

Cistos ovoides.

Hospedador: Scolopendra (sp.)

Habitat: intestino.

Procedencia: Rio de Janeiro.

Epoca: Novembro de 1917.

Ao nosso colega Sr. ALVIM TEIXEIRA DE AGUIAR, e ARTHUR PAULO DE SOUZA MARTINS que nos forneceram - material para o estudo deste protista, sinceros agradecimentos.

\section{Gregarina brasiliensis PINTO, 1918. (Est. 2 Figs. 30 e 31).}

Lomprimento total do protozoxirio 92 micra; idem do protomerito 38 micra; largura do mesmo 30 micra; idem do deutomerito 35 micra; comprimento do nucleo 20 micra; largura do mesmo 10 micra, cariosoma 4 micra.

Relações entre tais dimensðes: rełação entre o comprimento do protomerito e o comprimento total da gregarina $1: 2,4$; relação entre a largura do deutomerito e a do protomerito $1: 1,1$; relação entre a largura do protomerito e o comprimento do mesmo $1: 1,2$.

Protomerito ovo-cilindrico, geralmente incurvado, a máior parte das vezes obliquamente implantado na extremidade anterior do deutomerito (Fig. 31). Deutomerito piriforme. Epicito de espessura bastante pronunciada quer no protomerito quer no deutomerito. Endocito de constituição granulosa tanto no protomerito como no deutomerito. Cistos e esporos não observámos. Nucleo, oval com cariosoma de morfologia diversa. No nucleo observam-se granulações de cromatina de varios tamanhos e irregularmenle dispostas.

Movimento. Quando se observa esta gregarina, nota-se lógo um movimento bastante pronunciado. O deslocar do protista se faz sempre em um só sentido, isto é, para frente.

Diagnostico diferencial. Esta greg:t- rina assemelha-se com a Gregarina grisea ELLIS, e com a Actinocephalus stelliformis AIME' SCHNEIDER. Da primeira se distingue por possuir nitida constricção do septo e não formar sizigia, e tambem pela disposição da extremidade posterior do protomerito.

Da Actinocephalus stelliformis A. Sch., se diferencia por possuir esta gregarina um epimerito que se conserva por muito tempo, e, ainda pela disposição do septo que na referida gregarina é sempre plano.

A Gregarina brasiliensis conserva sempre a mesma fórma do deutomerito. - que não se observa na Actinocephalus stelliformis A. Sch., que pela disposição do deutomerito apresenta tres variedades: piriforme, longa e sub-esferica.

Hospedador: Scolopendra (sp.)

Habitat: intestino.

Procedencia: Rio de Janeiro.

Epoca: Outubro de 1917.

Ao Dr. SEVERINO LESSA, que gentilmente nos forneceu o material para o estudo desta gregarina, aqui deixamos os nossos agradecimentos.

\section{Gregarina elegans PINTO, 1918.} (Est. 2 Fig. 37).

Dimensões do protozoario e as suas partes componentes: comprimento total 75 micra; idem do protomerito 10 micra; largura do mesmo 24 micra; idem do deutomerito 32 micra; comprimento do nucleo 12 micra; largura do mesmo 5 micra; cariosoma 2 micra.

Relações entre essas dimensões: relação entre o comprimento do protomerito e o comprimento total da gregarins 1:7.5; relação entre a largura do deutomerito e a do protomerito 11,2 ; relação entre a largura do protomerito para - comprimento do mesmo 1:2.4.

Protomerito curto achatado no sentido antero-posterior, possuindo no centro uma formação oval cujo maior diametro é disposto no sentido transversal. 
Não podemos identificar esta formação a um nucleo por isso que não apresenta estrutura peculiar á deste orgão. Na extremidade anterior do protomerito existe uma serie de filamentos.

Deutomerito piriforme. Epicito bastante espesso no deutomerito, fino em o protomerito. Endocito de constituição kranulosa e de côr parda no protomerito; no deutomerito ele tambem possue identica estrutura, sendo entretanto. de côr mais escura. Nucleo. Em preparados fixados pelo sublimado alcool de SCHAUDINN e corados pela hematoxilina ferrea. segundo HEIDENHAIN, observámos melhor a estrutura desta parte do protista. O nucleo apresenta a fórma de uma pera, possuindo cariomembrana bastante pronunciada e de espessura desigual. No interior dele e na parte anterior existe um cariosoma de fórma um tanto oval. Não conseguimos ver centriolo no interior ou fóra do cariosoma. Linina irregularmente disposta no interior do nucleo.

Movimentos. Esta gregarina possue mobilidade, deslocando-se sempre para a frente e não possuindo movimentos de retorno como se observam na Gregarina aragãoi. observar.

Cistos e sizigias não conseguimos

Hospedador: Scolopendra (sp.)

Habitat: intestino.

Procedencia: Rio de Janeiro.

Epoca: Setembro de 1917.

\section{Gregarina watsoni PINTO, 1918.}

(Eet. 2 e 3 Figs. 39-43).

Dimensões "dos exemplares por nós observados: fórmas em evolução desde 54 micra de comprimento até esporontes medindo 350 micra de extensão.

Dimensðes de um exemplar possuindo ainda o epimerito (Fig. 41): comprimento total 130 micra; idem do epimerito 12 micra; largura do mesmo 20 micra; comprimento do protomerito 25 micra; largura do mesmo 38 micra; largura do deutomerito 40 micra. Nucleo com $10 \mathrm{mi}$ cra de diametro, cariosoma com 4 micra.

Dimensões do esporonte: comprimento total 350 micra; idem do protomerito 52 micra; largura do mesmo 104 micra; idem do deutomerito 152 micra. Nucleo com um diametro de 30 micra, cariosoma com 10 micra.

Dimensões da sizigia 570 micra; comprimento do primito 300 micra; idem do sateilte 270 micra.

Epimerito globular, não possuindo movimentos apreciaveis. Protomerito com pequenas variações de estrutura, conforme se considerar um protozoario ainda joven, em evolução ou já no estado de esporonte. Nas fórmas jovens, esta parte do protista se apresenta mais ou menos cilindro-conica e possue pequena constricção na parte média. Nos estadios intermediarios (possuindo epimerito) o protomerito apresenta a fórma um tanto piramidal, observando-se constantemente uma constricção em sua parte média. Nos protomeritos dos esporontes, porém já não é observado este caracter, apresentando-se eles apenas com uma porção semi-globulosa de plasma. Os esporontes possuem um protomerito muito menor $\mathbf{e}$ não tem epimerito. O septo é mais ou menos plano em as fórmas jovens e intermediarias, e faltando nos esporontes.

Deutomerito piriforme nas gregarinas jovens; cilindrico, terminando em ponta romba nas fórmas intermediarias. Nos esporontes ele é completamente cilindrico. Movimentos. A gregarina movese em linha recta com certa rapidez, ás vezes porém ela estaciona por alguns segundos e, então, a parte posterior do deutomerito curvando-se sobre sua parte anterior, efetua movimentos de curvatura para a direita e para a esquerda, apresentando neste caso o deutomerito a fórma de um V. (Fig. 43).

Epicito bastante apreciavel nos esporontes e ausente nas fórmas jovens e 
intermediarias. Endocito de côr parda e de constituição granulosa no protomerito e no deutomerito, sendo mais claro na parte anterior do epimerito e no protomerito do esporonte. Nucleo com a fórma redonda, possuindo um grande cariosoma. Sizigia. Observámos alguns exemplares.

Ao Dr. ADOLPHO LUTZ que teve a gentileza de classificar o hospedador deste gregarina, e ao Dr. MAGARINOS TORRES que nos forneceu o material para este estudo, os nossos agradecimentos.

Hospedador: Omoplata normalis Germ. (Coleoptero).

Habitat: intestino.

Procedencia: Nictheroy.

Epoca: Abril de 1918.

Gregarina chagasi PINTO, 1918.

(Ests. 2 e 4 Figs. 38 e $56-60$ ).

Dimensões do protozoario: comprimento total 130 micra; idem do protomerito 35 micra; largura do mesmo 50 micra; idem do deutomerito 50 micra. Nucleo 10 micra. Relações entre essas dimensões: relação entre o comprimento do protomerito e o comprimento total $1: 3.6$; relação entre a largura do protomerito e a do deutomerito $1: 1,5$; relação entre a largura do protomerito para o comprimento do mesmo 1:1,5.

Protomerito de fórma sub-globular, achatado no sentido antero-posterior, conservando-se com esta morfologia desde os estadios jovens da gregarina. Deutomerito com a fórma cilindrica. Em os estadios jovens do protozoario, se estreita na parte posterior. A' medida que a gregarina evolue para esporonte, o deutomerito sofre alteração na sua morfologia e apresenta então, igual largura em toda a sua extensão. Nos estadios intermediarios o deutomerito apresenta-se com a fórma cilindrica, quasi sub-globular; depois, isto é, quando esporonte ele toma uma fórma alongada com achatamento na parte posterior.
Epicito com espessura uniforme, bastante flexivel. Em estadios jovens desta gregarina notámos na parte posterior do deutomerito, mionemas bem visiveis (Fig. 57). Endocito de côr amarela com granulacões esparsas no protomerito e no deutomerito. Em alguns espécimeas notámos uma faixa hialina na parte anterior do deutomerito (Fig.58).

Nucleo redondo com membrana nuclear ás vezes bem nitida e apresentando em alguns exemplares um cariosoma redondo e um pouco excentrico (Fig. 58).

Cistos. Observámos cistos que tem a fórma ovoide e com dimensões muito pequenas (Fig. 59 e 60). Esporos não conseguimos observar.

Movimentos. Gregarina com deutomerito flexivel no terço anterior, dando lugar a que a parte anterior do protista isto é, protomerito e pequena porção do deutomerito se dobre para a direita e para a esquerda (Fig. 56). Depois do protozoario efetuar tais movimentos, avança pequena porção em linha recta e estaciona por alguns segundos, havendo exemplares que se movem para diante e para traz.

Hospedador: Conocephalus frater Redt. (Gafanhoto).

Habital: intestino.

Procedencia: Manguinhos.

Epoca: Dezembro de 1917.

Ao Professor Dr. A. DA COSTA LIMA, da Escola de Agricultura, os nossos agradecimentos por haver classificado o hospedador desta especie de gregarina.

Gregarina aragãoi PINTO, 1918.

(Ests. 3, 4, 5 Figs. $44-47,50-55,61,02.72-78$ ).

Dimensões da gregarina: comprimento total 170 micra; largura do deutomerito 70 micra; comprimento do protomerito 30 micra; largura do mesmo $40 \mathrm{mi}$ cra; comprimento do epimerito 10 micra: largura do mesmo 10 micra. Sizigia: comprimento do primito 160 micra; largura do mesmo 160 micra; comprimento do satelite 150 micra; largura do mes- 
mo 140 micra; nucleo 12 micra de diametro; cariosoma 8 micra. Epimerito de fórma cilindrica com a extremidade arredondada, pouco movel, ás vezes deslocando-se para a direita ou para a esquerda.

Protomerito de morfologia variavel conforme o estado de evolução da gregarina, ou apresenta a fórma quasi hemisferica, (o que se nota nos estadios jovens do protista) ou se nos apresenta com a fórma cilindrica, arredondada na extremidade anterior.

Deutomerito de fórma oval, mais ou menos alongada. Epicito de espessura uniforme e bastante flexivel. Endocito claro hialino em alguns exemplares, tornando muitas vezes quasi invisivel a gregarina. Em outros exemplares o endocicito é de côr parda claro, tornando dest' arte bem visivel o protozoario. Em preparados corados pela hematoxilina ferrea (metodo de HEIDENHAIN) o endocito apresenta uma estruutura alveolar.

Nucleo (Fig. 78) redondo com membrana nuclear bem visivel. A maior parte das vezes o nucleo é central, entretanto vimo-lo na parte anterior do deutomerito e excentricamente. Em córtes de intestino fixados pelo sublimado alcool de SCHAUDINN e coloridos pela hematoxilina de HANSEN, pudémos observar melhor a estrutura do nucleo. Este apresenta um grande cariosoma. Comumente notámos um granulo cromatico em a zona do suco nuclear, quasi sempre muito proximo e ligado ao cariosoma por um delgado filamento ou véo de cromatina. ASTROGILDO MACHADO observou tais granulos no Schizocystis spinigeri MACHADO.

HARTMANN, PROWAZEK e MACHADO consideram estes granulos cromaticos como sendo centriolos saídos do cariosoma. O nucleo quasi sempre possue granulos volumosos de cromatina.

A fórma do cariosoma é quasi sempre redonda. Sizigias observámos algumas vezes (Fig. 61 e 62 ). A fig. 61 representa uma fórma de pre-encistamento das gregarinas. Esporos não conseguimos observar.

Movimentos e mudança de fórma. - Alguns exemplares tem movimentos rapidos para a frente, outros, para a direita ou para a esquerda. Nestes ultimos movimentos o protozoario dobra-se sobre si mesmo, e o endocito se condensa de tal maneira que a fórma da gregarina é bem diversa da normal (Fig. 51). Observámos espécimens desta gregarina que tomavam a fórma um tanto arredondada e moviam-se á maneira de um ciliado (Fig. 50), após alguns segundos a gregarina tomava uma fórma um tanto alongada e continuava a se deslocar para diante ou para os lados.

Ciclo evolutivo. Só conseguimos observar uma parte do ciclo evolutivo da Gregarina aragãoi embora tivessemos examinado grande numero de coleopteros hospedadores deste esporozoario. Não conseguimos ver os espóros nem os cistos em diversos gráos de maturidade, impedindo-nos deste modo a observação dos gametos, sua conjugação, copula, formação de zigoto, espóros e esporozoitos.

Apezar de possuir o hospedador um intestino extremamente fino, fisemos a fixação em sublimado alcool de SCHAUDINN, incluimo-lo em parafina e praticámos cótes seriados em toda a extensão. dele, colorindo-os pela hematoxilina ferrea de HEIDENHAIN.

O esporozoito de fórma alongada afasta os cilios da celula epitelial nela penetrando (Fig. 73); uma vez dentro da celula epitelial o esporozoito afasta - nucleo da celula parasitada e toma a fórma oval (Fig. 72). Em estado mais adiantado de evolução, o joven parasito apresenta dois segmentos (Fig. 75 ou os tres (Fig. 74) localisando-se o seu nucleo no deutomerito. Depois de parasitar e de se desenvolver á custa da celula epitelial a gregarina abandona-a (Fig. 75), ficando por algum tempo preso a ela pelo epimerito (Fig. 76). Muitas vezes as gregarinas permanecem por algum tem- 
po assim presas e em se destacando da celula parasitada deixam o epimerito á ela apenso, ou então o protozoario se destaca e conserva-o por muito tempo.

Hospedador: Systena (sp.) pequeno coleoptero.

Habilat: intestino.

Procedencia: Manguinhos.

\section{Gregarina légeri PlNTO, 1918. (Est. 6 Fig. 86).}

Dimensðes do protozoario: comprimento total 290 micra; idem do protomerito 60 micra; largura do protomurito na parte anterior 70 micra; idem na parte posterior 50 micra; largura do deutomerito 60 micra; idem do deutomerito na parte dilatada 80 micra. Nucleo 50 micra por 30 micra.

Relações entre essas dimensões: relação entre o comprimento do protomerito e o comprimento total $1: 4,8$; relação entre a largura do protomerito e a do deutomerito $1: 1$; relação entre a largura do protomerito para o comprimento do mesmo 1:1.

Protomerito curto, cilindrico e mais largo na parte anterior; aí esta parte da gregarina apresenta vestigios do epimerito que se destacou.

Deutomerito cilindrico, terminando na parte posterior por uma porção dilatada em esfera, o que torna a Gregarina légeri facil de se distinguir de todas as outras.

Epicito de duplo contorno, não apresentando estriação aparente. Endocito granuloso de côr parda no protomerito e no deutomerito. Nucleo de fórma oval e situada na parte posterior dilatada do deutomerito. Cistos e espóros não conseguimos observar.

Esta especie de gregarina só encontrámos uma vez parazitando Stylopyga americana.

Hospedador: Stylopyga americana. (Barata).

Habitat: cavidade intestinal.
Procedencia: Rio de Janeiro. Léme. Epoca: Dezembro de 1917.

Ao Dr. ABELARDO MELLO que nos forneceu material para o estudo desta gregarina, os nossos agradecimentos

Botriopsis claviformis PINTO, 1918.

(Est. 4 Figs. 63-67).

Dimensôes. Observámos exemplares que mediam de 100 a 200 micra de comprimento. Largura do deutomerito $50 \mathrm{mi}$ cra; comprimento do protomerito $35 \mathrm{mi}$ cra; largura do mesmo 70 micra.

Epicito bem desenvolvido. Protomerito achatado no sentido antero-posterior, sempre muito mais largo que o deutomerito, formando com este um conjunto claviforme. Na maior parte dos exemplares notamos na parte anterior do protomerito uma zona clara desprovida de granulações.

Deutomerito mais largo na parte anterior, terminando posteriormente sempre por uma extremidade romba. Nesta parte da gregarina existem em quasi todos os exemplares, manchas de fórmas diversas. Estas manchas são de constituição granulosa, sempre porém, mais claras que o resto do endocito do deutomerito.

No protomerito nunca notámos tais manchas. O septo é sempre concavo e penetra no protomerito á maneira de uma cunha. O nucleo póde ser redondo, oval ou em fórmas de alteres. Cistos desconhecidos.

Hospedador: Larva de Aeschnida

(Odonata).

Habitat: intestino.

Procedencia: Manguinhos.

Epoca: Julho de 1918.

Ao Dr. ADOLPHO LUTZ que nos forneceu material para o estudo deste protista os nossos agradecimentos.

Monocystis perforans PINTO, 1918.

(Ests. 4, 5 e 6 Figs. 68-71, 94-100).

FORMACÃO DOS ESPOROZOITOS OBSERVADA IN VITRO.-Como a pre- 
sente monocistidea apresentasse grande quantidade de espóros, resolvemos observar a formação dos esporozoitos, o que conseguimos com relativa facilidade. $\mathrm{Pa}-$ ra apreciarmos tal fenomeno tomámos cistos contendo sómente espóros em diversos gráos de maturidade e misturamo-los com solução fiziologica a 8,50/oo, depois colocámos a mistura em uma camara humida na temperatura de $21^{\circ}$. Meia hora depois de havermos observado o espóro, lentamente formou-se o primeiro esporozoito; no fim de uma hora haviam se formado seis esporozoitos com espaço de tempo mais ou menos igual para cada um deles. Não podemos contar os outros dois esporozoitos por estarmos trabalhando com material em estado fresco, porém, em preparações ulteriormente coradas pelo metodo de HEIDENHAIN ou pelo hemalume-eosina, verificámos possuir o referido espóro, oito esporozoitos.

Espóro. O Monocystis perforans possue espóros biconicos providos de uma parede resistente com polos semelhantes, regulares e lisos (angioespóro). Endoesporo de fórma oval com oito esporozoitos em fórma de crescente, tendo cada um deles um nucleo centralmente colocado. Os esporozoitos medem cinco micra de comprimento por um micron de largura. O espóro mede 24 micra de extensão por 7.5 micra de largura. $O$ endoesporo: tem 14 micra de comprimento por 6,5 micra de largura. Na parte média do espóro observámos a fresco e em preparaç̃es coradas, uma pequena formação redonda contornada por sete outras, formando dest'arte o conjunto uma figura de rosacea. Esta figura de rosacea representa o reliquat do espóro.

O corpo do Monocystis perforans apresenta-se com fórmas diversas, predominando entre elas a cilindrica. As gregarinas podem ser observadas isoladas ou em sizigias. Os esporontes são vistos a olho nú e medem 1.200 micra por 800 micra de largura. Os nucleos dos esporontes que medimos tinham $150 \mathrm{mi}$ cra de comprimento por 50 micra de largura; cariosoma de fórma arredondada e irregularmente disposto no interior do nucleo. O protoplasma desta gregarina é grandemente vacuolado em toda a extensão do protista.

Agradecemos ao Professor CARLOS MOREIRA, do Museu Nacional a finesa de haver classificado o obligoqueto hospedador do Monocystis perforans.

Hospedador: Glossoscolex wiengreeni Mchlsn. (Michocuçú).

Habitat: testiculo.

Procedencia: Rio de Janeiro (Gavea). Epoca: Julho de 1918. 


\section{Explicação das figuras.}

Fig. 1 Espóro da Fonsecaia polymorpha PINTO, 1918

Fig. 2 Fórma redonda da Fonsecaia polymorpha.

Fig. 3 Fórma redonda da Fonsecaia polymorpha vista de cima para baixo.

Figs. 4-10 Fórmas em evolução da mesma gregarina. $\mathrm{Na}$ fig. 7 os mionemas são visiveis sómente na metade anterior do deutomerito.

Fig. 110 mesmo exemplar da fig. 17 (esporonte) que se contraio e tomou a fórma da fig. 11.

Fig. 12 Fonsecaia polymorpha em fórma de vaso; na parte anterior do deutomerito vem-se os mionemas.

Figs. 13-16 Fonsecaias evoluindo para esporontes.

Fig. 17 Esporontes de Fonsecaia polimorpha. O protomerito é pequeno em relação ao deutomerito. $\mathrm{O}$ epimerito não possue granulações. Na parte média do esporonte existe uma faixa clara devido ao afastamento das granulações do endocito. Mionemas muito nitidos colocados na parte média do esporonte. Nucleo em fórma de rosacea contendo um carisoma.

Figs. 18-24 Fórmas jovens da Gregarina magalhãesi PINTO, 1918. O epimerito é bem visivel em todos os exemplares, com excepção do da fig. 23 que não possue tal formação. Nas figuras 20 22 o epimerito é ameboide e como tal muda de aspéto conforme os movimentos efetuados pelo protomerito.

Figs. 25-28 Fórmas da mesma gregarina evoluindo para esporonte.

Fig. 29 Esporonte da Gregarina $m a-$ galhãesi. Nucleo invisivel. O epi- cito fórma dobras e penetra em um terço do endocito.

Figs. 30 e 31 Gregarina brasiliensis PINTO, 1918. Coloração pela hematoxilina ferrea (metodo de HEIDENHAIN). A fig. 30 representa uma gregarina em repouso e a fig. 31 um exemplar em movimento. Nucleo de fórma oval na fig. 31 e liptico em a fig. 30 . Dentro do nucleo vêm-se massas de cromatina de tamanhos e fórmas diversas, fortemente coloridas pela hematoxilina ferrea. Linina em fórma de granulos.

Fig. 37. Gregarina elegans PINTO, 1918.

Fig. 32-36 Stenophora lutzi PIN'TO, 1918. O exemplar da fig. 32 perdeu o epimerito, os demais possuem'no. Todos os exemplares são nucleados, o da fig. 36 mostra um cariosoma redondo.

Figrs. 38, 56-60 Gregarina chagasi PINTO, 1918. As figuras $57 \mathrm{e}$ 58 são fórmas jovens da gregarina; a fig. 38 representa uma fórma em evolução, o septo é invisivel. A fig. 56 representa o esporonte. Fig. 59 e 60 cistos desta gregarina. Na fig. 57 vêmse mionemas na parte terminal do deutomerito. Na fig. 58 ve-se uma faixa hialina colocada na parte anterior do deutomerito.

Fig. 39-43 Gregarina watsoni PINTO, 1918. Fig. 39 fórma joven da gregarina. Na parte anterior do protomerito existe uma mancha escura. A fig. 40 representa uma fórma em movimento, vendo-se o epimerito e o protomerito dobrados sobre o deutomerito. Fig. 42 sizigia da gregarina. A fig. 41 mostra o epimerito, o protomerito e o deutomerito da Gregarina watsoni.

Fig. 43 Esporonte da Gregarina wa- 
tsoni. O deutomerito está recurvado para um dos lados.

Fig. 49 Stenophora polydesmi (Lak) WATSON.

Figrs. 44-47, 50-55, 61 e 62. Gregarina aragãoi PINTO, 1918. A fig. 55 representa um exemplar muito pequeno desta gregarina visto com grande aumento, o protista possue os tres segmentos. Fig. 45 e 46 exemplares em evolução possuindo epimerito. A fig. 50 representa um exemplar de Gregarina aragãoi visto de cima para baixo, nesta posição o protozoario roda para a direita e depois para a esquerda como se fosse um ciliado. A fig. 51 representa um exemplar visto em posição obliqua e dirigindo-se para a mesma posição do exemplar da fig. 50.

Figrs. 52 e 53 Exemplares desta gregarina evoluindo para esporonte.

Fig. 54 Esporonte da Gregarina aragãoi (exemplo tipico de uma gregarina.)

Figs. 61 e 62 . A fig. 62 representa uma sizigia desta gregarina e a Fig. 61 outra em estado de pre-encistamento; 0 satelite da fig. 61 está com o protomerito achatado.

Figs. 63-66 Bothriopsis claviformis PINTO, 1918.

Fig. 67 Cistos de Bothriopsis claviformis.

Fig. 68 Cisto de Monocystis perforans PINTO, 1918. com gametos.

Fig. 69 Cisto da mesma gregarina com espóros.

Fig. 70 Exemplar de Monocystis perforans.

Fig. 71 Sizigia de Monocystis perforans.

Figs. 72-76 Fórmas endocelulares da Gregarina aragãoi.
Fig. 73 Esporozoito da Gregarina aragãoi penetrando em umacelula epitelial.

Fig. 72 Esporozoito evoluindo para a formação de uma gregarina.

Fig. 74 Gregarina aragãoi já formada e parasitando uma celula epitelial.

Fig. 75 Gregarina aragãoi saindo de uma celula epitelial.

Fig. 77 Cisto da Gregarina aragãoi.

Fig. 78 Nucleo da Gregarina aragãoi desenhado com oc. 3 e obj.im. $1 / 12$.

Junto do nucleo vê-se o centriolo contornado por uma zona clara.

Figs. 79-80 Stenophora umbilicata PINTO, 1918.

Fig. 81 Stenophora tenuicolis PINTO, 1918.

Figs. 82-85 Stenophora lutzi PINTO, 1918.

Fig. 84 Exemplar muito joven de Stenophora lutzi.

Fig. 86 Gregarina légeri PINTO, 1918.

Fig. 87 Fórma joven de Stenophora cunhai PINTO, 1918.

Fig. 88 Esporonte de Stenophora cunhai.

Fig. 89 Esporonte de Stenophora cruzi PINTO, 1918.

Fig. 90-92 Stenophora viannai PINTO, 1918. A fig. 92 representa o protomerito e o deutomerito com uma papila.

Fig. 93 Nucleo de Protomagalhaensia serpentula (MAGALHÃES, 1900) desenhado com oc. 6 comp. e obj. 1,8. A fig. 48 representa uma sizigia desta gregarina.

Fig. 94-100 Espóros de Monocystis perforans PINTO, 1918. desenhados com oc. 4 e obj. im. 1/12. No centro do espóro vê-se a figura de uma rosacea constituida pelo reliquat do espóro. Da fig. 95 em diante vêm-se os esporozoitos dentro do espóro. 
A.

1. AWERINZEW, S. Beobachtungen uber die Vorgange der Schizogonie bei Gregarinen aus den Darm von... In Archiv f. Protistenkunde. Iena. Vol. 16 pag. 71.

B.

2. BRASIL, L. 1905 La reproduction des Gregarines monocystides. In Arch. zool exp. Paris. (4) t. III pag. 17 e t. IV pas, 69.

3. BRASIL, L. 1905/6 Nouvelles recherches sur la reproduction des gregarines monocystides. In Arch. zool. exp. (4) t. IV pp. 69-99 pl. IX-X.

4. BRASIL, L. 1908 Le genere Doliocystis. In C. R. Acad. Sc. Paris. t. 146.

5. BRASIL, L. 1909 Documents sur quelques sporozoaires d'Annelides. In Archiv f. Protistenkunde. Iena. Vol. XVI pag. 107.

6. BOLDT. 1910 In den S er enden Monocystideen, In Sch. der Phy. oknomischen r.esell. zu Konisgsberg. pp. 55-66.

7. BOLDT, MARTIN Zwwei riris Gregarinenarten aus Octalasium complanatum Ant, I) :gés. In zool. Anzeiger. Vol. 36 pp. 289-293.

8. BUETSCHLI, Klassen u Ord. des Thier-Reichs. (Frster Band) Protozoa. pp. Dr. O. $1880 / 82 \quad 513$ pl. XXXII-XXXVII.

9. BRUCH 1850 L:inige l emerkungen uber die Gregarinen. In Zeit. u. Zool. Vol, It pag. 110.

10. BEDDARD 1888 Note on a new gregarine. In Proc. Zool. Soc. Londres pp. 354.

11. BERNDT, 1902 Beitrage zur Kennntnis der im Darme der Larve von Tenebrio Dr. ARTHUR molitor lebenden Gregarinen. In Archiv f. Protistenkunde, Iena. Vol. l. pp. $375-420$ pl. XI--XIII.

\section{C.}

12. CALKINS, 1915 Microtaeniella clymenellae, a new genus and new species of GARY N. $\quad$ colonial gregarines. In Biol. Bull. of the Marine Biol Lab. Vol. XXIX N. lpp. 46-49.

13. CAUllieRY e Le parasitisme des gregarines. In C. R. Acad. Sc. Paris t. CXXXII MESNIL pag. 220 e C. R. Soc. Biol. Paris. t. III-4. pag. 84.

14. CAULlERY, M. Sur une gregarine celomique presentant dans son cycle évolutif et MESNIL 1898 une phase de multiplication asporulee (Gonospora longissima). In C. R. Soc. Biol. Paris L. 65 e C. R. Acad. Sc. Paris t. CXXVI-3 pag. 262.

15. CAULLERY \& Sur un mode particulier de division nucléaire chez les grégariMESNIL 1900 nes. In Ach. Anat. micros. t. III.

(1) Bibliografia até 1919, epoca que entregámos o trabalho para impressão. 
16. CECCONI, J. 1902 De la sporulation de la Monocystis agilis Stein In Arch. Anat micros. t. V. pp. $122-140$ pl. V.

17. COMES, 1907 Untersuchungen uber den Chromidialapparat der Gregarinew Dr. S. In Archiv i. Prot. Iena. Vol. $10 \mathrm{pp}$. $416-440 \mathrm{pl}$. XIX-XX.

18. CRAWLEY, The progressive movement of Gregarines. In Proc. of the Acad. H. 1902 of Nat. Sc. of Philadelphia. Vol. LIV. Part I. pp $4-19$ pl. I-II.

19. CRAWLEY, H. 1903

List of the Polycistid Gregarines of the United States. In Proc. of the Acad. of Nat. Sc. of Philadelphia. Vol. LV. part. I pp. $41-58 \mathrm{pl}$. I-III.

20. CRAWLEY, $H$.

The Polycistid Gregarines of the United States (second contri. bution) In Proc. of the Acad. of Nat. Sc. of Philadelphia. Vol. LV part. III pp. 632-644. pl. XXX. 21. CUENOT, L. 1897 Evolution des gregarines coelomiques des grillon domestique.
In C. R. Ac. Sc. Paris. Vol. CXXV. pag. 52. 22. CUENOT, L. 1899 Sur la pretendue conjugaison des Gregarines. In Arch. de Biol.
t. VII.

23. CUENOT, L. 1902 Recherches sur l'évolution et la conjugaison des gregarines. In Archives de Biol. Vol. 17 pp. 581-652.

24. CUNNINGHAM, On Kalpidorhyncus arenicolae a new Gregarine parasitic in
J. T. 1007 . Arenicola ecaudata. In Archiv fur Protist. Iena. Vol 10 pp. $199-215$ pl. VI-VII. 24a. CUNHA, R, ${ }_{1919}$ Sobre a Gregarina neo-brasiliensis AL. CUNHA, 1919. Trabalho
DE AL. ${ }_{1919}$ apresentado á Faculdade de Medicina de apresentado á Faculdade de Medicina de Bello Horizonte (Minas Geraes. Brasil).

\section{D.}

25. DOBELL, C. C. 1909

Chromidia and the Binuclearity Hypotheses. In Quarterly Jour. of Micr. Sci. Londres. Vol. 53 pp. 295-298.

26. DOFLEIN, Dr. F. Lehrbuch der Protozoenkunde.

27. DOGIEL, VALENTIN 1906

Beitrage zur Kenntnis der Gregarinen. I. Cystobia chiridotae nor. sp. In Arch. f. Protist. Iena Vol. VII pp. 106-130 pl. III.

28. DOGIEL, V. 1909 Beitrage zur Kenntnis der Gregarinen. III. Uber die Sporocysten der Colom-Monocystidae. In Arch, f. Protist. Iena,

29. DOGIEL, V. 1910 Beitrage zur Kenntnis der Gregarinen. IV Callynthrochlamys phronimae Frez. u. a. m. In Arch. f. Protist. Iena. Vol. 20 pp. $60-78$.pl. VII. 30. DRZEWIECKI, Uber vegetative Vorgange im Kern und Plasma der Gregarinen.
Dr. WS. 1907 II Sotmatophora coronata nov. gen. (Monocystis coronata Hesse) In Arch. f. Protist. Iena. Vol. 10. pp.
216-216. 
31 DUFOUR, LEON

\section{7}

Recherches sur quelques Entozoaires et larves parasites des insectes Orthoptéres et Hyménoptéres. In Ann. Sc. nat. p. zool. (s. 2), t. 7 pp. 5-20.

\section{E.}

32. ELLIS, MAX. M. A descriptive List of Cephaline Gregarine of the New World. In Trans. of Amer. Micr. Society. Vol. XXXII. N. 4 pp. 259-296. pl. XVII-XX.

33. ELLIS, M. M. 1918 Five polycistid Gregarines from Guatemala. In Zool. Anzeiger. Leipzig. Vol. 39 pag. 680-89.

34. ELLIS, M. M. 1913 Three gregarines from Louisiana. In Zool Anzeiger. Leipzig. e Berlin Vol. 42 pp. 200-2.

35. ELLIS, M. M. 1914 An acanthosporid gregarine from North Amer. dragonfly nymaphs. In Trans. Amer. Micr. Soc. Illinois. Vol. XXXIII N. 4 pp. $215-222$.

F.

36. FRENZEL, J. 1855 Uber einige in Seethieren lebende Gregarinen. In Arch. f. mikr. anat. Vol. XXIV pp. 545-588 pl. XXV-XXVI.

37. FUCHS, Dr. G. Die Naturgeschichte der Nematoden u. einiger anderer Parasiten im Darm des Hylobius abietis L., Clepsidrina hylobii n. sp. In Zool. Jahrbucher. Iena. Vol. 38 pp. 203/4.

G. 38. GABRIEL, B. $1880 \mathrm{Zur}$ classification der Gregarinen. In Zool. Anz. Vol. 3 pp.

H. 39. HARTMANN, Das System der Protozoen. In Archiv f. Protist. Iena. Vol. 10
PROF. Dr. MAX. 1906.

40. HASSELMANN, Contribuição para o estudo das gregarinas. IN BRASIL-MEDICO. PROF. Dr. GUSTAVO $\quad 1918$ Rio-N. 32 de 10-8--918. (Ancora lutz n. sp.)

41. HASSELMANN, Um novo esporozoario pertencente á ordem Gregarinida BuePROF. Dr. G.

1918 tschli, 1882 21-9-918.

42. HASSELMANN, Novos esporozoarios da ordem Gregarinida Buetschli, 1882. In PROF. Dr. G. BRASIL-MEDICO, N. 40 de 5-10-1918. 43. HASSELMANN, Novos esporozoarios da ordem Gregarinida Buetschli, 1882. In
PROF. Dr. G. 1918 BRASIL-MEDICO N. 47 de 23-11-918.

44. HASSELMANN, Novos esporozoarios da ordem Gregarinida Buetschli, 1882. In PROF. Dr. G. 1918 BRASIL-MEDICO N. 44 de 2 de Novembro de 1918. 
45. HENEGUY 1887 Formation des spores de la Gregarine du lumbric. In C. R. Soc Biol. (3) t. 4 pp. $439-442$.

46. HENEGUY 1888 Formation des spores de la Gregarine du lumbric. In Ann. Microgr., t. I.

47. HESSE, ED. $1909 / 10$ Contribution a l'étude des Monocystidées des oligochetes. In Arch. zool. exp. (5) t. 3 pp. 27-30 1 pl. VII.

48. ISHII, SHIGEMI 1915

On a new Polycistid Gregarine, Spirosoma caudata nov. gen., e nov. sp., from a Diplopod. In Annotationes Zoologicae Jaionenses. Tokyo. Vol. IX pp. 7-9.

K.

49. KEILIN, D. 1918 On the occurence of a cephalina Gregarine, Leidyana tine? n. sp. im lepidopterous larve. In Parasitology. Londres. Vol. 10 N. 3 pp. 406-410.

50. KISSKALT, 1910 Pratikum der Bakteriologie und Protozoologie.

K. und HART-

MANN, Dr.

MAX

51. KÖLLIKER,

A.

Beitrage zur Kenntniss niederer Thiere. In Zeitschrift f. Wissenschaftliche Zoologie. Vol. I pp. $1-37$ pl. I-III.

52. KUSCHAKE- Beobachtungen uber vegetative, degenerative und germinative WITSCH, SERGIUS $\quad 1907$ Vorgange bei den Gregarinen des Mehlwundarms. In Arch. f. Protist. Iena. Supl. I pp. 202-249 pl. XII-XVII e 12 fig. no texto.

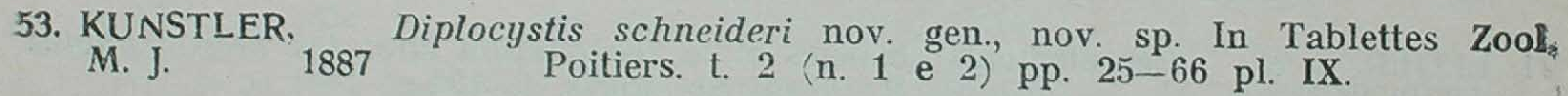

\section{L.}

54. LABBÉ, AL- Das Thierreich (Sporozoa).

\section{PHONSE} 55. LABBE, \& RA- Gregarine nouvelle parasite des Maldawiens. In Bul. Soc. Zooß
COWITZA $1897 \quad$ France t. XXII. 56. LANKESTER, Gregarines. In Quart. Jour. Microsc. Sc. t. III pp. 83-96
E. RAY $1863 \quad$ pl. VII. pl. VII.

57. LANKESTER, Notes on the Gregarinide. In Trans. Micr. Soc. Vol. XIV n. E. R.

s. pag. $27 \mathrm{pl}$. V fig. 89 .

58. LAVERAN, A. et Sur quelques particularités de l'évolution d'une Gregarine, et̂. MESNIL, F. 1900

la réation de la cellule hote. In C. R. Soc. Biol. Paris. t. 52 pp. $554-7$. 59. LÉGER, 1892 Recherches sur les Grégarines. In Tabl. Zool. Poitiers. t. 3
LOUIS
N. 1 e 2 pp. $1-182$ pl. I-XXII. N. 1 c 2 pp. 1-182 pl. I-XXII.

60. LÉGER, L. 1893 Sur une Gregarine nouvelle des Acridiens d'Algerie. In C. R., Acad. Sc. Paris. Vol. 117 pp. 811-13. 
61. LF́GER, L. 1894 Sur une nouvelle Gregarine de la familie Dactilophoridae. In. C. R. Acad. Sc. Paris, liv. 118 pp. 1285-88.

62. LÉGER, L. 1897 Contributioon á connaissance des sporozoaires parasites des Echinodermes. In Bull. Scient. France of Belgique. t. XXX.

63. LÉGER, L. Les Gregarines et I'epithelium intestinal. In C. R. Acad. Sc. Paris. t. $\mathrm{CXXX}$.

64. LÉGER, L. Sur la morphologie des elements sexuels chez les Gregarines Stylorhynchidae. In C. R. Acad. Sc. Paris. t. CXXXII.

65. LÉGER, L. et Notes biologiques sur les grillions. In Arch. Zool. exper. Paris. DUBOSCQ, O. 1899

(3) t. VII pag. XXXV.

66. LEEGER, L. 1900 Sur les Gregarines des Diptéres (Stylocystis) In. Ann. Soc Ent. France. IXVIII-3 pag. 526.

67. LÉGER, L. et Les Gregarines et l'epithelium intestinal. In C. R. Acad. Sc. DUBOSCQ.

O.

1900

Paris. Vol. 130 pag. 1556.

68. LÉGER, L. et Sur les premiers states du développement de quelques PolyDUBOSCQ, O. 1901 cistides. In C. R. Acad. Sc. Paris. t. CXXXIII pag 439.

69. LÉGER, L. 1901 Les elements sexueles et la copulation chez les Stylorhynchus. In C. R. Acad. Sc. Paris. t. CXXXIII.

30. LÉGER, L. et Les Gregarines e l'epithelium intestinal chez les trachéates. DUBOSCQ, O. 1902 In Arch. Parasitologie. Paris. t. 6. pp. 377-473 pl. II-VI.

71. LEGER, L. et Les elements sexueles et la fecondation chez les Pterocephalus. DUBOSCQ, O. 1902

72. LÉGER, L. et DUBOSCQ, $\mathrm{O}$, 1903

Recherches sur les myriapodes de Corse et leurs parasites. Avec la description des diplopodes por H. W. Brolemann. In Arch. Zool. expe. Paris. t. l. pp. 307-356. 73. LÉGER, L. et Note sue le developpement des Gregarines Stylorhynchidae
DUBOSCQ, O. 1903 et Stenophoridae. In Arch. zool. exp. (4) t. I. N. 6. pp. XIV-XCV.

74. LÉGER, L. 1903 La reproduction sexueé chez Plerocephalus. In Arch. zool. exp. Paris. t. 1 . 75. LÉGER, $L_{\text {, et }}$ La reproduction sexueé chez Pterocephalus. In Arch. zool. exp
DUBOSC, O. 1903

76. LÉGER, L. et La reproduction sexueé chez Pterocephalus. In Arch. zool. expDUBOSCQ, O. 1903

Paris. t. l. (4) pp. CXLI-CXLVII.

77. LÉGER, L. et DUBOSCQ, O. 1903

Aggregata vagans n. sp. Gregarine gymnosporeé. In Arch. zool. exp. Paris. (4) t. 1. N. 9 pp. CXLVII-CLI. 
78. LÉGER, L. et Nouvelles recherches sur les gregarines et l'epithelium intestinal. DUBOSCQ, O. 1904

In Arch. f. Protistenkunde. Iena. t. IV.

79. LÉGER, L. et Sur l'evolution des Gregarines gymnosporeés des crustaces.

O. In C. R. Acad. Sc. Paris. t. 142 pag. 1225.

80. LÉGER, L. . 1906 Etude sur Taeniocystis mira Léger. Gregarine métamerique. In Arch. f. Protist. Iena. Vol. VII pp. $307-329$ pl.

81. LÉGER, L. et DUBOSCQ, O. 1907

L'evolution des Frenzelina n. gen. gregarines intestinales des crustacés técapodes. In C. R. Sc. Paris. Vol. 145 pp.

82. LÉGER, L. et
DUBOSCQ, O. 1909

Etudes sur la scxualité chez les gregarines. Arch. f. Protist. Iena. vol. 17 pp. 17-134 pl. I-V.

83. LÉGER, L. 1909 La reproduction sexueé chez les Actinocephalidae. In C. R. Acad. Sc. Paris. T. 148 pp. 190-193. 84. LÉGER, L. et Porcspura nephropis n. sp. In C. R. Soc. Biol. Paris. t. 78 pp.
DUBOSCQ, O. 1915

85. LEVDIG

1851 Uber Psorospermien und. Gregarinen. In Muller's Archiv pp. 221-274 tav. 8. 86. LIEBER-
KUHN $1854 / 55$ $\begin{gathered}\text { Evolution des Gregarines. In Mem. cour. Acad. roy. de Bel- } \\ \text { gique, t. } 26\end{gathered}$

87. LIEBERKUHN 1854

Evolution des Gregarines. In Acad. roy. de Belgique. t. XXVI des mem. couronnés, 1854.

88. LUHE, Dr. M.1904 Bau und Entwicklung der Gregarinen. In Arch. f. Protist. Iena. Vol. 4 pp. 88-198.

M.

89. MAGALHÃES, Notes d'helminthoiogie brésilienne. In Arch. de Parasitoligie. PROF. P.

S. de 1900

Paris. t. III pp. 34-69.

90. MARTIIS,

Dr. L. C. 1911

Descrizione d'una nuova Gregarina policistidea parasita d'un oligochete. In Arch. f. Protist. Iena. Vol. 23 pp. 247-252 pl. 11.

91. MARTIIS, Dr. L. C. 1911

Le monocistides e loro fenomini riproduttivi. In Arch. f. Protist. Iena. Vol 23 pp. 205-246.

92. MERCIER,

L. $1912 / 13$

Monographie D'Uradiophora cuenoti, Grégarine parasite du tube digestive de la Caridine. In Arch. zool. exp. et gen. (5) vol. 10 pp. 177-202 pl. XIII-XIV.

93. MINCHIN, 1917 An intr. to the Study of the protozoa. London.

94. MINGAZZINI, P. 1893

Contribito alla conoscenza dgli Sporozoi. In Ric. Lab. Anat. norm. Univers. Roma t. III. 
95. MOROFF, Dr. T. 1908

96. MOROFF, Dr. T. 1908

97. MULSOW, KARL
Untersuchungen uber Coccidien. Gregarininfektion. In Arch. f. Protist. Iena. Vol. VIII pp. 23-25.

Die bei den Cephalopoden vorkommenden Aggregatarten etc. In Arch. f. Protist. Iena. Vol. 11 pp. 1-224.

Uber Fortpflanzunserscheinunger bei Monocystis rostrata $\mathrm{n}$. 1911 sp. In Arch. f. Protist. Iena. Vol. 22 pp. 20-55 pl. 2-6.

\section{N.}

98. NOWLIN, N. The intracellular developement of a Gregarine Frenzelina amand SMITH, INEZ 1917 pelisca n. sp. In The Jour. of Parasitology. Vol. IV N. 2 pp. $81-88$.

99. NUSBAUN, J.1993 Schaudinnella heulae mihi. In Zeitschr. f. wiss. Zool. t. LXXV pp. 281-307 pl. XXII.

P.

100. PAEHLER, $\mathrm{F}$. 1904

Uber die Morphologie, Fortpflanzung und Entwicklung vor Gregarina ovata. In Arch. f. Protist. Iena. Vol. 4 pp. 64-87 pl. V e VI.

101. PFEFFER, E.

Untersuchungen uber die Gregarinen im Darm der Larve von 1910 Tenebrio molitor. In Arch. f. Protist. Iena. Vol. pp. 107-118 pl. III.

102. PIXELL-GOO- On the Life History of the Sporozoa of Spatangoids, with DRICH, H. 1916 Observations on some Allied Forms. In Q. Jour. of Micrs. Sc. Vol. 61 pp. 81-104 pl. 8 .

103. POCHE, F. 1913 Das System der Protozoa. In Arch. \&. Protist. Iena. Vol. 30 pp. 125-310.

104. PRENANT, A. 1903

Questions relatives aux cellules musculaires. In Arch. zool. exp. Paris. (4) t. I pp. C-CIV.

105. PROWAZEK, Zur Entwicklung der Gregarinen. In Arch. f. Prot. Iena. Vol. I Dr. S. von 1902 pp. 297-305 pl. IX.

106. PINTO, CEZAR Sobre as eugregarinas parasitas dos artrópodes brasileiros. NotaFERREIRA 1918 prévia. In BRASIL-MEDICO. N. 7 de 16-2-918.

107. PINTO, C. $\mathrm{F}$. 1918

108. PINTO, C. F. 1918

109. PINTO, C. F. 1918 Sobre as eugregarinas parasitas dos atrópodes brasileiros. Segunda nota-prévia. In BRASIL-MEDICO. N. 8 de 23-2-918.

110. PINTO, C. F. 1918

Sobre as eugregarinas parasitas dos atrópodes brasileiros. QuinSobre as eugregarinas parasitas dos atrópodes brasileiros. Terceira nota-prévia. In BRASIL-MEDICO. N. 9 de 2-3-918.

Sobre as eugregarinas parasitas dos atrópodes brasileiros. Quarta nota-prévia. In BRASIL-MEDICO. N. 12 de 23-3-918.

111. PINTO, C. $F$. 1918 ta nota-prévia. In BRASIL-MEDICO. N. 13 de 30-3-918;

Sobre as eugregarinas parasitas dos atrópodes brasileiros. Sextał nota-prévia. In BRASIL-MEDICO. N. 15 de 13-4-918. 
112. PINTO, C. F.

1918 Sobre as eugregarinas parasitas dos atrópodes brasileiros. Setima nota-prévia. In BRASIL-MEDICO. N. 26 de 29-6-918.

113. PINTO, C. F.

1918

Sobre as eugregarinas parasitas dos atrópodes brasileiros. Oitava nota-prévia. In BRASIL-MEDICO. N. 30 de 27-7-918.

114. PINTO, C. $F$. 1918 Estudos sobre gregarinas. Nona nota-prévia. In BRASIL-ME-
DICO. N. 35 de $31-8-918$.

115. PINTO, C. $F$.

1918 Estudos sobre gregarinas. Decima nota-prévia. In BRASIL- ME-
DICO. N. 38 de $21-9-918$.

176. PINTO, C. $F$.

1918

117. PINTO,

C. F.

Estudos sobre gregarinas. Decima-segunda nota-prévia. In BRA-
SIL-MEDICO. N. 41 de $12-10-918$.

Estudos sobre gregarinas. Decima-primeira nota-prévia. In BRASIL-MEDICO. N. 39 de 28-9-918.

S.

118. SCHELLAK, Uber die Entwicklung und Fortpflanzung von Echinomera hisC. 1904 pida (A. Sch.) In Arch. f. Protist. Iena. Vol. IX pp.

119. SCHNEIDER, Sur quelques points de l'histoire du genero Gregarina. In Arch. AIME C. J. 1873 zool. exp. Paris. t. II. pp. 515-533 pl. XX. 120. SCHNEIDER, Polydystides. In Arch. zool. exp. Paris. t., 4 pag. 514 e (1) t.
$\begin{array}{lll}\text { A. C. J. } 1875 & 4 \text { pp. } 4-597 .\end{array}$ 121. SCHNEIDER, Contribution a l'histoire des Gregarines. In Arch. zool. exp.
A. C. J. $1875 \quad$ Paris. t. IV pp. $493-604$ pl. XVI-XXIII.

122. SCHNEIDER, Etudes sur le développement des grégarines. In Tabl. zool. A. C. J. 1885 Poitiers. T. I. N. 1 e 2 pp. $10-24$ pl. IV-VI.

123. SCHNEIDER, Grégarines nouvelles ou peu connues. In Tabl. zool. Poitiers. A. C. J. $1885 \quad$ I. Nos. 1 e 2 pp. $25-30$. pl. $\mathrm{X}-\mathrm{XI}$.

124. SCHNEIDER, Grégarines nouvelles ou peu connues, (deuxiéme article). In
A. C. J. 1885
Tabl. zool. Poitiers. T. I. Ns. 3 e 4 pp. $90-103$. 125. SCHNEIDER,
A. C. J. 1885 Tonference sur le parenté des coccidies et des gregarines. In

Tabl. Zool. Poitiers. T. I. Ns. 3 e 4. pp. 104-120. pl.

126. SCHNEIDER, Etudes sur le developpement des grégarines (deuxiéme article). A. C. J. 1896 In Tabl. zool. Poitiers. T I I N.St 3 e 4 ipag. 81. pl. XVIII.

127. SCHNEIDER, Grégarines nouvelles ou peu connue. In Tabl. zool. Poitiers.

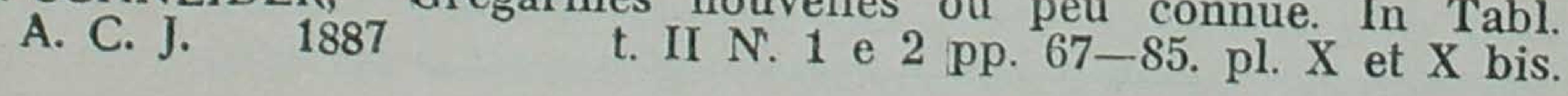

128. SCHNEIDER,
$\begin{aligned} & \text { A. C. J. } 1892\end{aligned} \quad$ Sur le genero Pilocephalus. In Tabl. Zool. Poitiers. T. 2 Ns.
3 e 4 pp. $199-207$ pl. XXXI 129. SCHNEIDER, Contribution a l'histoire des Gregarines des Invertebrés de
A. C. J. Paris et de Roscoff. In Arch. de Zool. exp. (1) t. 4 pag. 493 e Arch. de Zool. exp. t. 10 pag. 423. 
130. SCHNITZLER, Uber die Fortpflanzung von Clepsidrina ovata. In Arch. $\mathrm{f}$. H. $\quad 1905 \quad$ Protist. Iena. Vol. VI pp. 309-333. pl. XVI-XVII.

131. SCHEWIA- Uber die Ursache... Bewegung der Gregarinen. In Zeitsch. f. KOFF 1894 wiss. Zool.VII pag. 340.

132. SIEDLECKI, Contrib. à l'étude des changements cellulaires provoques par les M. 1901 gregarines. In Arch. Anat. microsc. t. 4 pag. 87.

133. SOKOLOW, Liste des gregarines décrites depuis 1889. In Zool Anzeiger. B. $1911 \quad$ Leizig. t. 38. pp. 277-95.

134. SOKOLOW, Studien uber Physiologie der Gregarinen. In. Arch. f. Protist. B. 1912 Iena. Vol. 27 pp. 260-314.

135. STUARDT 1871 Uber den Bau der Gregarinen. In Bull. Ac. Imp. de Sci. de St. Petersbourg. t. 15 pp. $497-502$. est. 15 fi. $1-5$.

136. SWARCZEWS- Chromidien bei den Gregarinen. In Biol. Centralbl. Vol. 32 pp. KY, B. $1912 \quad 435-445$ e $449-458$.

T.

137. TRÉGOUBOFF, Sur les Grégarines des Balanes. In. Arch. zool. exp. Paris. (5) G. $\quad 1912 \quad$ t. $10 \mathrm{pp}$. LIII-LXI.

W.

138. WOODCOCK, On Cystobia irregularis (Minch.) and allied "neogamous». GreH. M. 1904 garines. In Arch. zool. exp. (4) t. II; Notes et Revues no. 8.

139. WATSON, Studies on gregarines. In Illinois Biological Monographs. Vol. M. E. 1916 II N. 3.

140. WATSON, Three new gregarines from marine crustacea In Jour. of PaM. E- 1916 rasitology. N. 2 pp. 129-136. pl. I.

141. WATSON, Observations on Polycistid Gregarines from Orthopodes. In Jour. M. E. 1916 of Parasitology. Vol. III N. 2 doc. 1916 pag. 65.

142. WATSON, The Developement of Gregarines and their Relation the Host (KAMM)

M. E. 1918 Tissues: in Stenophora lactaria M. E. Watson. In Jour. of Parasitology. t. II de 3-3-917. pp. 124-130 2 pl.

143. WATSON,
(KAMM) M. E.

New gregarines from coleoptera. In Jour. of Parasitology. Urbana (Illinois) N. 4 Vol. IV pp. 159-163, June de 918 